\title{
Bottom-up nonempirical approach reducing search space in enzyme design guided by catalytic fields Supporting Information
}

\author{
Wiktor Beker, W. Andrzej Sokalski
}

Wroclaw University of Science and Technology, Wyb. Wyspianskiego 27, 50-370 Wroclaw, Poland

e-mail: Wiktor.Beker@wp.eu e-mail:Andrzej.Sokalski@pwr.edu.pl 


\section{Contents}

1 Dynamic catalytic field $\vec{\Delta}_{d}\left(\vec{r}_{i}\right) \quad 2$

2 ONIOM calculations $\quad 2$

3 MMPBSA calculations $\quad 4$

4 Summary of mutant data $\quad 6$

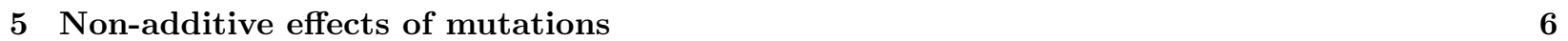

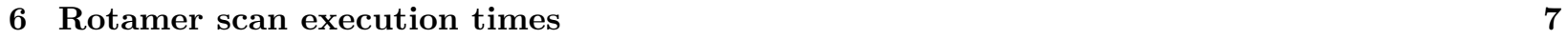




\section{Dynamic catalytic field $\vec{\Delta}_{d}\left(\vec{r}_{i}\right)$}

Further information about best catalytic enviroment may be provided by $\Delta_{S}$ gradient, called dynamic catalytic field:

$$
\vec{\Delta}_{d} \stackrel{\text { def }}{=} \operatorname{grad} \Delta_{S}=\vec{F}_{T S}-\vec{F}_{S}
$$

Oryginal interpretation, basing on derivation published in [1], is that this vector field provides an insight into optimal movement of enviromental charges to 'follow' reaction coordinate, thus lowering the barrier more efficiently than static surroundings. However, such properties can be hardly controlled in molecular design and yet may recquire more sophisticated treatment, which makes application of so interpreted $\vec{\Delta}_{d}$ rather cumbersome. Intersingly, this differential vector field may be interpreted in two more ways.

Geometry optimisation. As a gradient of $\Delta_{S}$, it could be used to optimize catalyst geometry toward best agreement with catalytic field.

Catalytic dipoles. While $\Delta_{S}$ provides information about optimal positions of catalytic charges, $\vec{\Delta}_{d}$ means the same for location of catalytic dipoles. This interpretation is particularly interesting, since lowering reaction barrier by a set preorganised dipoles seems to be very common in biocatalysis.

Proof of the latter of above interpretations is rather simple. Assuming enviromental dipoles $\left\{\vec{\mu}_{i}\right\}_{\text {env }}$ instead of charges $\left\{q_{i}\right\}_{e n v}$, interaction energy with TS (or substrate, respectively) becomes

$$
E_{I E}(T S) \approx-\sum_{i \in e n v} \vec{\mu}_{i} \cdot \vec{F}_{T S}\left(\vec{r}_{i}\right)
$$

and DTSS energy:

$$
E_{I E}(T S)-E_{I E}(S) \approx-\sum_{i \in e n v} \vec{\mu}_{i} \cdot\left(\vec{F}_{T S}-\vec{F}_{S}\right)\left(\vec{r}_{i}\right)=-\sum_{i \in e n v} \vec{\mu}_{i} \cdot \vec{\Delta}_{d}\left(\vec{r}_{i}\right)
$$

having minimal value when $\vec{\mu}_{i}$ are located in places with possibly big $\left|\vec{\Delta}_{d}\left(\vec{r}_{i}\right)\right|$, their directions are aligned with $\vec{\Delta}_{d}\left(\vec{r}_{i}\right)$ and are possibly big by themselves.

This interpretation of dynamic catalytic field was tested on the ketosteroid isomerase(KSI) [2] and protein kinase systems (PKA) [3]. Dynamic catalytic field was found to agree perfectly with hydrogen bond donors in KSI oxyanion hole (Fig. S2). In PKA, similar agreement is found with neutral residues showing the best contribution to DTSS (like Gly55 - Figure S1). However, with more complex residues like aspargine, the interpretation is more difficult and not clear.

\section{ONIOM calculations}

First, structures of reactants (RS), transition state (TS) and IRC reaction path in KE07 were obtained with ONIOM method implemented in Gaussian09 [4]. Benzoizoxazole and part of Glu 101 residue $\left(\mathrm{O}_{\varepsilon 1}, \mathrm{O}_{\varepsilon 2}\right.$, $\mathrm{C}_{\delta}, \mathrm{C}_{\gamma}, \mathrm{H}_{\gamma 1}, \mathrm{H}_{\gamma 2}$ ) were assigned to QM layer (B3LYP/6-311+G**) with link atom (hydrogen) for broken $\mathrm{C}_{\gamma}-\mathrm{C}_{\beta}$ bond. The rest of the protein together with surrounding sphere of water molecules were modelled by AMBER94 force field; the interaction between QM and MM layer was included via mechanical embedding scheme. Coordinates of atoms further than 17 Åfrom QM layer were frozen during all calculations. Input files were prepared with TAO package [5] supported by a Python script written in order to calculate connectivity data. Force field parameters for 5-nitrobenzoisoxazole were obtained with AmberTools2012 [6]. As refitting charges of QM region may be inportant in ONIOM studies [7], two cycles of corresponding procedure were performed for TS structure, which led to rather minor changes in its geometry (see Figure S4). 


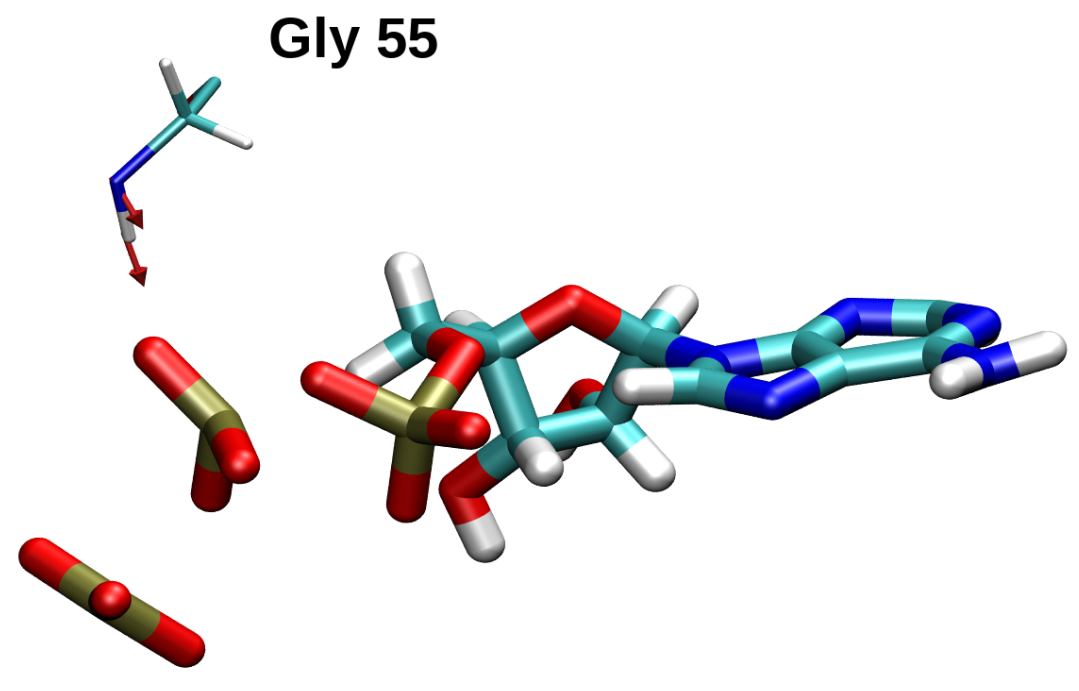

Figure S1: $\vec{\Delta}_{d}\left(\vec{r}_{i}\right)$ in PKA, represented by vectors colored according to the magnitude of dynamic catalytic field (blue-small, red- large).

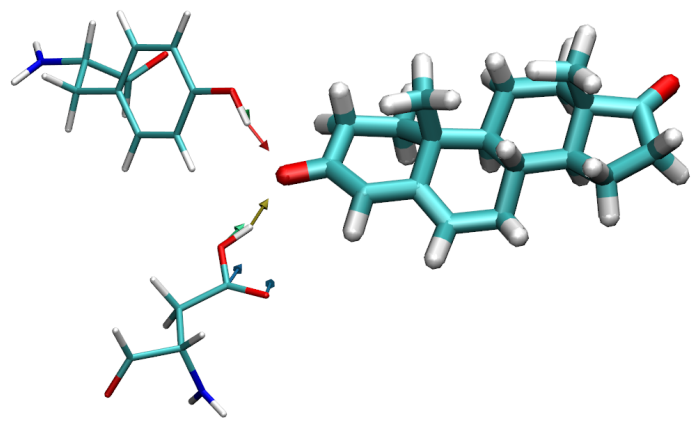

Figure S2: Dynamic Catalytic Field in KSI,represented by vectors colored according to the magnitude of $\vec{\Delta}_{d}\left(\vec{r}_{i}\right)$ (blue-small, red- large). 
a)

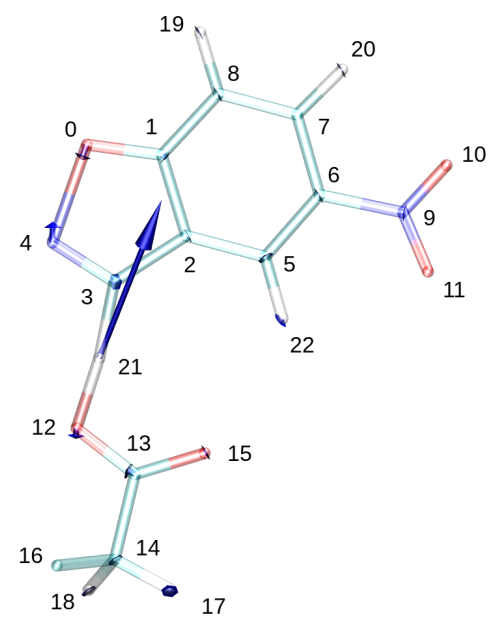

b)

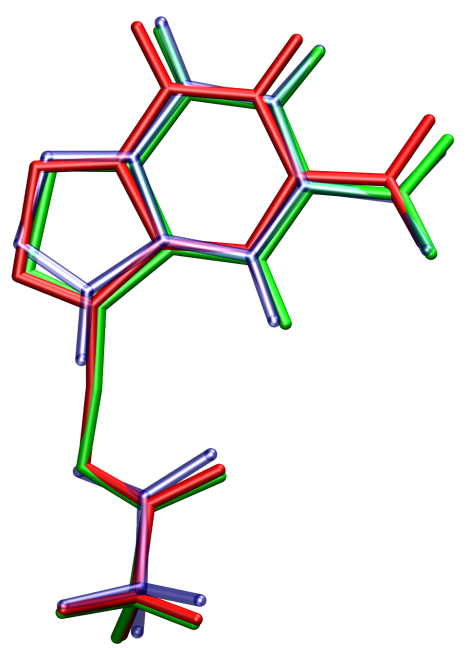

Figure S3: Transition State in KE07.

a) Imaginary frequency $\left(-940.6819 \mathrm{~cm}^{-1}\right)$. Main contributions comes from $\mathrm{H}_{21}$ movement along $\mathrm{O}_{12}-\mathrm{C}_{3}$ axis and opposite displacements of $\mathrm{O}_{0}$ and $\mathrm{N}_{4}$, corresponding to reaction process.

b) Comparison of structures of reactant, TS and TS after two cycles of charge reffiting [7]. Blue- reactant; red- TS before chage refitting procedure; green- final TS structure.

\section{$3 \quad$ MMPBSA calculations}

In order to justify the usage of differential charges, consider termodynamic cycle presented in Figure S4. Then, reaction barier in solvated enzyme may be expressed as in equation (4). On the other hand, binding energy (as calculated here with g.mmpbsa) can be written as the sum of enzyme-ligand interaction energy and the difference between solvation energy of the complex with those of enzyme and ligand (5).

$$
\begin{aligned}
B_{E, \text { solv }} & =B_{\text {int }}+\Delta E(E \cdot T S)-\Delta E(E \cdot S)+\Delta G_{\text {solv }}(E \cdot T S)-\Delta G_{\text {solv }}(E \cdot S) \\
& =B_{\text {int }}+E_{D T S S}+\Delta \Delta G_{\text {solv }} \\
\Delta G_{\text {bind }}(L) & =\Delta E(E \cdot L)+\Delta G_{\text {solv }}(E \cdot L)-\Delta G_{\text {solv }}(E)-\Delta G_{\text {solv }}(L)
\end{aligned}
$$

Then, taking into account that solvation energy of a set of point charges may be expressed in terms of system electrostatic potential in solvent and vacuum, and the fact that linearized Poisson-Boltzmann equation allows to represent electrostatic potential as a superposition of potentials of components (provided that geometry and distribution of dielectric constant is kept the same), $\Delta G_{\text {solv }}(E \cdot L)$ may be decomposed according to equations (7)-(8).

$$
\begin{aligned}
\Delta G_{\text {solv }}(E \cdot L) & =\frac{1}{2} \sum\left(\phi_{\text {solv }}-\phi_{\text {vac }}\right) q_{i}=\frac{1}{2} \sum \Delta \phi q_{i} \\
\phi & =\phi^{E}+\phi^{L} \\
\sum \Delta \phi q_{i} & =\sum_{j \in E} \Delta \phi q_{j}+\sum_{k \in L} \Delta \phi q_{k} \\
& =\sum_{j \in E} \Delta \phi^{E} q_{j}+\sum_{k \in L} \Delta \phi^{E} q_{k}+\sum_{j \in E} \Delta \phi^{L} q_{j}+\sum_{k \in L} \Delta \phi^{L} q_{k}
\end{aligned}
$$




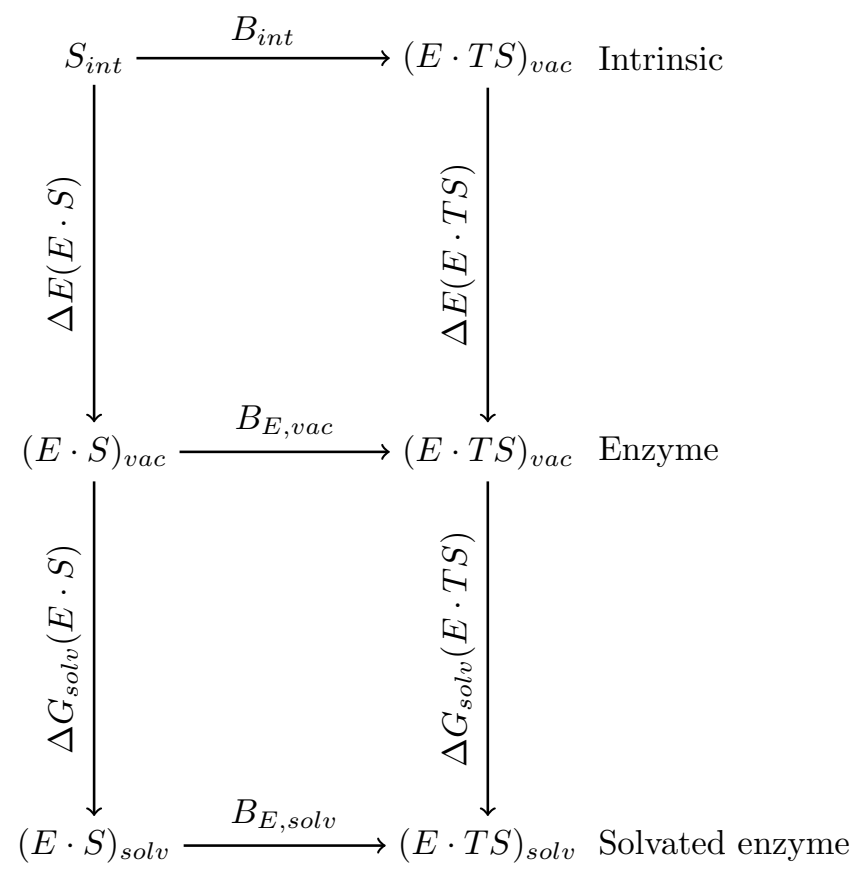

Figure S4: Thermodynamic cycle of one-step reaction.

Having that decomposition, the $\Delta \Delta G_{\text {solv }}$ from equation (4) may be re-written as (9), keeping in mind that we assumed the same geometry and dielectric distribution in substrate and TS.

$$
\begin{aligned}
2\left(\Delta G_{\text {solv }}(E \cdot T S)-\Delta G_{\text {solv }}(E \cdot S)\right) & =\sum_{k \in L} \Delta \phi^{E}\left(q_{k}^{T S}-q_{k}^{S}\right)+\sum_{j \in E}\left(\Delta \phi^{T S}-\Delta \phi^{S}\right) q_{j} \\
& +\sum_{k \in L}\left(\Delta \phi^{T S} q_{k}^{T S}-\Delta \phi^{S} q_{k}^{S}\right)
\end{aligned}
$$

Note that the first two sums in (9) can be evaluated with differential charges, while the last 'self-contribution' of reactants cannot. Let's apply the same decomposition to difference of solvation energies in equation (5); in order to do this, let's mark the potentials in $\Delta G_{\text {solv }}(E)$ and $\Delta G_{\text {solv }}(L)$ (with $L \in\{T S, S\}$ ) with subscript 0.

$$
\begin{aligned}
2\left(\Delta G_{\text {solv }}(E \cdot L)-\Delta G_{\text {solv }}(E)-\Delta G_{\text {solv }}(L)\right) & =\sum_{j \in E}\left(\Delta \phi^{E} q_{j}-\Delta \phi_{0}^{E} q_{j}\right)+\sum_{k \in L} \Delta \phi^{E} q_{k}+\sum_{j \in E} \Delta \phi^{L} q_{j} \\
& +\sum_{k \in L}\left(\Delta \phi^{L}-\Delta \phi_{0}^{L}\right) q_{k}
\end{aligned}
$$

Comparing eqeuations (10) and (9), one may see that they will correspond to each other $\left(\Delta G_{\text {solv }}(E \cdot T S)-\Delta G_{\text {solv }}(E \cdot S)\right.$ with solvation energy contribution to binding a ligand with differential charges) under two conditions: vanishing of 'protein-only' term (first sum in equation 10) and neglect of the 'ligand-only' term (the last sum). This justifies removal of 'self contribution' of QM region to DTSS energy calculated from differential charges in implicit solvent. The assumption of approximately constant protein solvation energy upon binding of ligand may be overcome by taking the difference of binding energies of TS and substrate, which provides a DTSS value shifted by the difference of solvation energies of sole reactants, which is common for all mutants (11).

$$
\Delta G_{\text {bind }}(T S)-\Delta G_{\text {bind }}(S)=E_{D T S S}+\underbrace{\Delta G_{\text {solv }}(E \cdot T S)-\Delta G_{\text {solv }}(E \cdot S)}_{\Delta \Delta G_{\text {solv }}(E \cdot L)}+\underbrace{\Delta G_{\text {solv }}(T S)-\Delta G_{\text {solv }}(S)}_{\text {common for all mutants }}
$$

The same conclusions are obtained with generalized Born model, which in this system provides reasonably accurate description of solvation energy. 


\section{Summary of mutant data}

Table S1: Directed evolution of KE07 [8]. Charged amino acids were marked with color corresponding to their formal charge (blue-negative, red- positive).

\begin{tabular}{|l|l|c|c|c|c|c|c|c|c|c|c|c|c|c|}
\cline { 3 - 11 } \multicolumn{1}{l|}{} & \multicolumn{10}{c|}{ Residue } \\
\hline Enzyme & $\mathrm{k}_{\text {cat }}$ & 7 & 12 & 19 & 77 & 84 & 86 & 102 & 123 & 146 & 202 & 207 & 224 & 229 \\
\hline KE07 & 0.018 & Ile & Val & Lys & Phe & His & Phe & Ile & Gln & Lys & Gly & Met & Asn & Phe \\
R2-11/10D & 0.0213 & Ile & Val & Glu & Phe & His & Phe & Ile & Arg & Thr & Arg & Met & Asp & Phe \\
R3-I3/10A & 0.206 & Gln & Val & Lys & Phe & His & Leu & Ile & Gln & Thr & Arg & Met & Asp & Ser \\
R4-1E/11H & 0.699 & Asp & Val & Lys & Phe & His & Phe & Ile & Gln & Glu & Arg & Met & Asp & Phe \\
R5-10/3B & 0.49 & Asp & Met & Lys & Phe & His & Phe & Ile & Gln & Lys & Arg & Met & Asp & Phe \\
R6-3/7F & 0.6 & Asp & Val & Glu & Phe & His & Phe & Ile & Gln & Thr & Arg & Met & Asp & Phe \\
R7-1/3H & 0.76 & Asp & Leu & Lys & Ile & Tyr & Phe & Ile & Gln & Lys & Arg & Thr & Asp & Phe \\
R7-10/11G & 1.37 & Asp & Met & Thr & Ile & His & Phe & Phe & Gln & Lys & Arg & Met & Asp & Phe \\
\hline
\end{tabular}

\section{Non-additive effects of mutations}

Another example of analysis of non-additive effects of mutations has been performed for Lys146 and Arg202. Scan over CAMM rotamer library has been performed in two ways: with inclusion of the mutual repulsion of residues and without. Each time, a structure with optimal energy in reactant state (that is the interaction of all three moyeties: QM region, R202 and K146) was chosen and rotamers with short contacts with surroundings were excluded. As can be seen, the DTSS contribution of Arg202 is in both cases the same, since it's closer to reactants; however, the DTSS of Lys146 as it took a more distant conformer due to repulsion with Arg202.

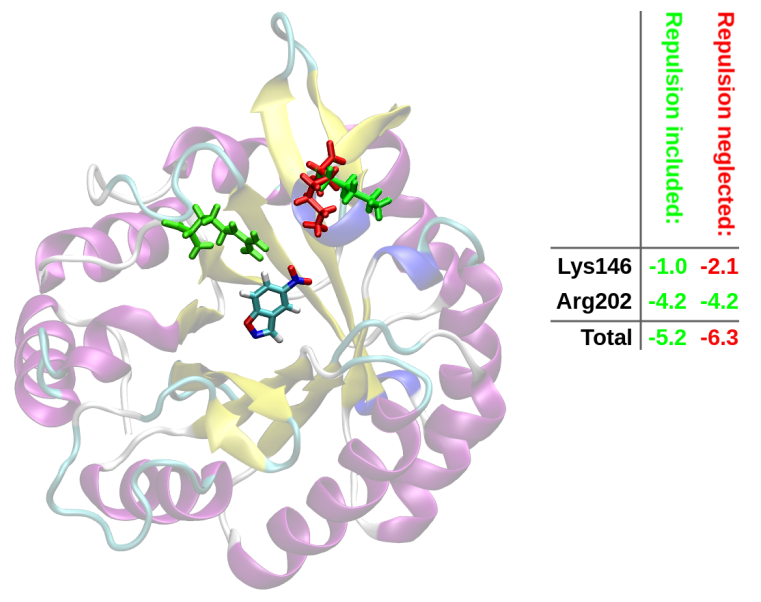

Figure S5: Activation barrier lowering DTSS (in $\mathrm{kcal} / \mathrm{mol}$ ) resulting from double rotamer scan of R202 and K146 with and without inclusion of their mutual repulsion 


\section{Rotamer scan execution times}

Table S2: Execution time of algorithm for exploration of rotamer configurational space. Test was prepared on a single CPU for $\mathrm{P}_{6}$, involving residues Asp7, Glu19, Lys146, Arg202 and Asp224. Interaction energy table was calculated from RESP charges. Initial configuration space was constructed from non-coliding rotamer combinations. Multiscan- brute force search over thus defined space; Dead End Elimination (DEE)-search over a space restricted by the Goldstein single criterion' DEE with threshold- DEE over space restricted by softened Goldstein criterion ( $\epsilon=0.005$ a.u.)

\begin{tabular}{|l|r|r|r|}
\cline { 2 - 4 } \multicolumn{1}{c|}{} & Multiscan & DEE & DEE with threshold \\
\hline Size of configurational space & 6257088 & 216 & 20160 \\
\hline Task & \multicolumn{3}{|c|}{ Execution time [s] } \\
\hline Loading rotamers to the structure & \multicolumn{3}{|c|}{18.336} \\
\hline Calculation of the energy table & 0.000 & 0.490 & 0.011 \\
\hline Single rotamer elimination (Goldstein criterion) & 67.836 & 0.003 & 0.210 \\
Evaluation of configurational space & 96.183 & 28.840 & 29.466 \\
\hline Sum & 77.847 & 10.504 & 11.130 \\
Sum (calculations only) & $\mathbf{6 7 . 8 3 6}$ & $\mathbf{0 . 4 9 3}$ & $\mathbf{1 . 1 1 9}$ \\
Sum (calculations without energy table)
\end{tabular}


Table S3: Execution time of algorithm for exploration of rotamer configurational space. Test was prepared on a single CPU for $\mathrm{P}_{6}$, involving residues Asp7, Glu19, Lys146, Arg202 and Asp224. Interaction energy table was calculated from CAMM expansion (up to $R^{-5}$ term). Initial configuration space was constructed from non-coliding rotamer combinations. Multiscan- brute force search over thus defined space; Dead End Elimination (DEE)-search over a space restricted by the Goldstein single criterion' DEE with thresholdDEE over space restricted by softened Goldstein criterion $(\epsilon=0.005$ a.u. $)$.

\begin{tabular}{|l|r|r|r|}
\cline { 2 - 4 } \multicolumn{1}{c|}{} & Multiscan & DEE & DEE with threshold \\
\hline Size of configurational space & 6257088 & 240 & 24360 \\
\hline Task & \multicolumn{3}{|c|}{ Execution time [s] } \\
\hline Loading rotamers to the structure & \multicolumn{3}{|c|}{37.425} \\
\hline Calculation of the energy table & 0.000 & 1.523 & 1.918 \\
\hline Single rotamer elimination (Goldstein criterion) & 75.328 & 0.003 & 0.271 \\
Evaluation of configurational space & 177.085 & 103.283 & 103.946 \\
\hline Sum & 139.660 & 65.858 & 66.521 \\
Sum (calculations only) & $\mathbf{7 5 . 3 2 8}$ & $\mathbf{1 . 5 2 6}$ & $\mathbf{2 . 1 8 9}$ \\
Sum (calculations without energy table) & &
\end{tabular}

\section{References}

[1] W. A. Sokalski. Nonempirical modeling of the static and dynamic properties of the opitimum nvironment for chemical reactions. J. Mol. Struct.(THEOCHEM), 138:77-87, 1986.

[2] W. Beker, M. van der Kamp, A. J. Mulholland, and W. A. Sokalski. Rapid estimation of catalytic efficiency by cumulative atomic multipole moments: application to ketosteroid isomerase mutants. $J$. Chem. Theory. Comput., 13:945-955, 2017.

[3] P. Szarek, E. Dyguda-Kazimierowicz, A. Tachibana, and W. A. Sokalski. Physical nature of intermolecular interactions within camp-dependent protein kinase active site: Differential transition state stabilization in phosphoryl transfer reaction. J. Phys. Chem. B, 112(37):11819-11826, 2008.

[4] S. Dapprich, I. Komaromi, K. S. Byun, K. Morokuma, and M. J. Frisch. A new oniom implementation in gaussian98. part i. the calculation of energies, gradients, vibrational frequencies and electric field derivatives. J. Mol. Struct., 461-462:1-21, 1999.

[5] P. Tao and H. B. Schlegel. A Toolkit to Assist ONIOM Calculations. J. Comput. Chem., 31:2363-2369, 2010.

[6] D.A. Case, T.A. Darden, T.E. III Cheatham, C.L. Simmerling, J. Wang, R.E. Duke, R. Luo, R.C. Walker, W. Zhang, K.M. Merz, B. Roberts, S. Hayik, A. Roitberg, G. Seabra, v Swails, A.W. Götz, I. Kolossváry, K.F. Wong, F. Paesani, J. Vanicek, J. Wolf, R.M.and Liu, X. Wu, T. Brozell, S.R. Steinbrecher, H. Gohlke, Q. Cai, X. Ye, J. Wang, M.-J. Hsieh, G. Cui, D.R. Roe, D.H. Mathews, M.G. Seetin, R. Salomon-Ferrer, v Sagui, V. Babin, T. Luchko, S. Gusarov, A. Kovalenko, and P.A. Kollman. AMBER 12. University of California, San Fancisco, 2012.

[7] P. Tao, J. F. Fisher, Q. Shi, T. Vreven, S. Mobashery, and H. B. Schlegel. Matrix Metalloproteinase 2 Inhibition: Combined Quantum Mechanics and Molecular Mechanics Studies of the Inhibition Mechanism of (4-Phenoxyphenylsulfonyl)methylthiirane and Its Oxirane Analogue. Biochemistry, 48(41):9839-9847, 2009.

[8] O. Khersonsky, D. Rothlisberger, O. Dym, C. J. Jackson, D. Baker, and D. S. Tawfik. Evolutionary optimization of computationally designed enzymes: Kemp eliminases of the ke07 series. Journal of Molecular Biology, 396(4):1025-1042, 2010. 